\title{
Keyword Index Volume 17 (2013)
}

\author{
action mining \\ active learning \\ ANOVA \\ anti-rumor \\ API usage \\ association rule \\ Association Rule Mining (ARM) \\ association rules \\ automata induction \\ averaging method \\ bankruptcy prediction \\ behavior \\ behavior modeling \\ betweenness centrality measure \\ binary decision diagrams \\ biological networks \\ bipartite graphs \\ bispectrum \\ boolean tensor \\ bug detection \\ burstiness
}

Cardiovascular Autonomic Neuropathy

causal networks

causal rules

centrality indices

chemical descriptors

class imbalance

classification

classifier

click logs

closed pattern

closed set lattice

cluster analysis

clustering

collaborative filtering
1075

251

771

149

459

803

271, 965

943

93

295

423

439

1041

217

343

295

5

309

49

459

125

309

1075

1075

217

327

423

423, 531

309,1115

837

49

399

1057

93, 179, 1001

899 complex networks

5

complex systems $\quad 753$

complexity 981

computational 981

concentration inequalities $\quad 217$

constrained clustering $\quad 485$

copula 233

copy-paste code $\quad 459$

cost-sensitive learning 423

credit risk $\quad 1115$

cube 943

data mining $\quad 387,459,507,753,791,917,965$

data preprocessing 737

data streams 439

decision support systems $\quad 753$

dependence 233

diabetes 309

diffusion 149

distance evaluation $\quad 737$

dynamic graphs $\quad 71,125$

dynamic weighting 367

dynamical systems 439

EA 771

ECG 309

e-commerce 899

enews mining 125

ensemble learning $\quad 367,387$

entity ranking 837

entropy 309

evaluation 1001

evaluation of temporal text mining $\quad 125$

evolution of networks 27

evolutionary approach 295

evolving graph 49

expectation maximization $\quad 343$ 
feature extraction

feature importance

feature subset selection

feature weighting

filtering technique

formal concept analysis

frequent pattern mining

fuzzy inference

Genetic Algorithm (GA)

graph analysis

graph mining

graph sharpening

graph visualization

graphical text summarization

group association rules

growth function

higher order spectra

HRV

human motion sequences

hybrid filtering

incremental mining

information bottleneck

interestingness measures

internal link prediction

itemset

K-means

K-Medoids

knowledge discovery

label ranking

linked data

logic programs with annotated disjunctions

485,1023

1023

917

399

179

343

machine learning

$387,531,1115$

mapping to multidimensional optimal regions 981

Markov chain

439

maximal

917

mixed-type data

mobility patterns

model interpretation

model visualization multivariate time series

mutual information

93,1057

1001

251

295

791

387

1115

patterns

531

plane graphs $\quad 71$

predictive accuracy 1115

probabilistic inductive logic programming 343

probabilistic logic programs $\quad 343$

profit mining $\quad 899$

$\begin{aligned} 5 & \text { proft mining } \\ 943 & \text { programming rule }\end{aligned}$

progressive database $\quad 507$

progressive mining $\quad 507$

proximity measures 179

QSAR modeling 327

random projection $\quad 217$

randomized algorithms 217

ranking 217

ranking metrics 837

RDF graph 179

recommendation 1093

recommender systems $\quad 899$

reliability 943

responsiveness 1041

ROC 531

ROC analysis $\quad 531$ 
rule discovery

rumor

search method parameters

self training

semantic dimension

semantic trajectories

semi-supervised clustering

semi-supervised learning

sequence database

sequence labeling

sequential

sequential patterns

shape-restriction

signaling pathways

similarity

simulation

social network mining

social networks

solution existance theorem

source code mining

sparse rating

statistical analysis
49 statistical metrics of data sets

149 statistical relational learning

343

statistical significance

771

771 statistical test

943

367 structure-based features

1057

27 supervised clustering 1023

857 support vector regression

485

$251,367,387,399$

507

251

899

507

233

295

179

753

217

149,1041

981

459

1093

771 temporal text mining $\quad 125$

text categorization 367

threshold prediction $\quad 803$

trajectory data mining $\quad 857$

turning points 27

utility analysis 271

utility based data mining 271

utility emphasized trading rules 271

V.C. dimension $\quad 981$

video applications $\quad 71$

weighted frequent pattern mining

917 